\title{
Quantitative imaging of bilirubin by photoacoustic microscopy
}

\section{Yong Zhou, Chi Zhang, Da-Kang Yao, Lihong V. Wang}

Yong Zhou, Chi Zhang, Da-Kang Yao, Lihong V. Wang, "Quantitative imaging of bilirubin by photoacoustic microscopy," Proc. SPIE 8581, Photons Plus Ultrasound: Imaging and Sensing 2013, 85813A (4 March 2013); doi: $10.1117 / 12.2004394$

SPIE. Event: SPIE BiOS, 2013, San Francisco, California, United States 


\title{
Quantitative Imaging of Bilirubin by Photoacoustic Microscopy
}

\author{
Yong Zhou†, Chi Zhang†, Da-Kang Yao, Lihong V. Wang* \\ Optical Imaging Laboratory, Department of Biomedical Engineering, Washington University in \\ St. Louis, One Brookings Drive, St. Louis, MO 63130, USA \\ $\uparrow$ The authors contributed equally to this work. \\ * Address all correspondence to: LHWANG@,WUSTL.EDU
}

\begin{abstract}
Noninvasive detection of both bilirubin concentration and its distribution is important for disease diagnosis. Here we implemented photoacoustic microscopy (PAM) to detect bilirubin distribution. We first demonstrate that our PAM system can measure the absorption spectra of bilirubin and blood. We also image bilirubin distributions in tissuemimicking samples, both without and with blood mixed. Our results show that PAM has the potential to quantitatively image bilirubin in vivo for clinical applications.

Keywords: photoacoustic microscopy, photoacoustic spectroscopy, bilirubin, scattering medium.
\end{abstract}

\section{Introduction}

Determining the bilirubin level and distribution plays important roles in the diagnosis of jaundice and other diseases. ${ }^{1-9}$ However, current approach to measuring bilirubin concentration involves extracting blood, which is invasive; only the total serum bilirubin level can be monitored without any spatial information. Noninvasive monitoring methods mainly include diffuse reflectance spectroscopy ${ }^{10-13}$ and hyperspectral imaging. ${ }^{71}$ The former senses the overall concentration of bilirubin in the skin without spatial resolution, whereas the latter maps the relative bilirubin distribution without quantification. Therefore, we still lack a technique for quantitative imaging of bilirubin.

Over the past few years, photoacoustic microscopy (PAM) has shown its promising ability for both structural and functional imaging. ${ }^{15-20}$ In PAM, the object is irradiated by a short-pulsed laser. Following the absorption of light, a temperature rise generates an initial pressure rise, which is propagated as a photoacoustic wave. This photoacoustic wave is then detected by a focused ultrasonic transducer ${ }^{21}$ Because the initial pressure is proportional to the local energy absorbed, photoacoustic measurements with multiple optical wavelengths can provide spectral information of optical absorption. By far, PAM has shown its feasibility to detect many intrinsic contrasts in biological tissue, such as hemoglobin, ${ }^{22-23}$ DNA and RNA in nuclei, ${ }^{24}$ water, ${ }^{25}$ lipid, ${ }^{26}$ cytochrome $\mathrm{c},{ }^{27}$ and melanin. ${ }^{28,29}$

Here we show that PAM can quantitatively image the bilirubin distribution. Based on the absorption spectra, proper wavelengths were chosen to measure pure bilirubin samples and mixed bilirubin-blood sample. The pure and blood mixed bilirubin distributions in tissue-mimicking samples were imaged. We showed for the first time that bilirubin, both inside and outside the blood vessels, can be quantitatively imaged by PAM with a high accuracy.

\section{System and Materials}

We used a free-space transmission-mode PAM system for bilirubin detection, as shown in Fig. 1(a). ${ }^{27}$ Briefly speaking, the laser pulse with a wavelength tunable from $210 \mathrm{~nm}$ to $2600 \mathrm{~nm}$, was generated by an integrated diodepumped Q-switched laser and optical parametric oscillator system (NT242-SH, Ekspla). After being filtered by an iris with $2 \mathrm{~mm}$ aperture size (ID25SS, Thorlabs), reflected by a mirror (PF10-03-G01, Thorlabs), focused by a condenser lens (LA4380, Thorlabs), and filtered by a pinhole with $50 \mu \mathrm{m}$ aperture size (P50C, Thorlabs), the light beam was at last focused by an optical objective with 0.3 numerical aperture (NA). The excited photoacoustic waves

Photons Plus Ultrasound: Imaging and Sensing 2013, edited by Alexander A. Oraevsky, Lihong V. Wang, Proc. of SPIE Vol. 8581, 85813A - C 2013 SPIE · CCC code: 1605-7422/13/\$18 - doi: 10.1117/12.2004394 
were detected by a focused ultrasonic transducer. Each laser pulse yields a one-dimensional depth-resolved image (A-line) by recording the arrival times of photoacoustic signals. A two-dimensional (2D) scanning stage (PLS-85, MICOS) was used to hold the sample and provide lateral scanning. We rendered our result in the form of a 2D maximum amplitude projection (MAP) image, which was produced by projecting the maximum amplitude of the 3D image along the axial direction.

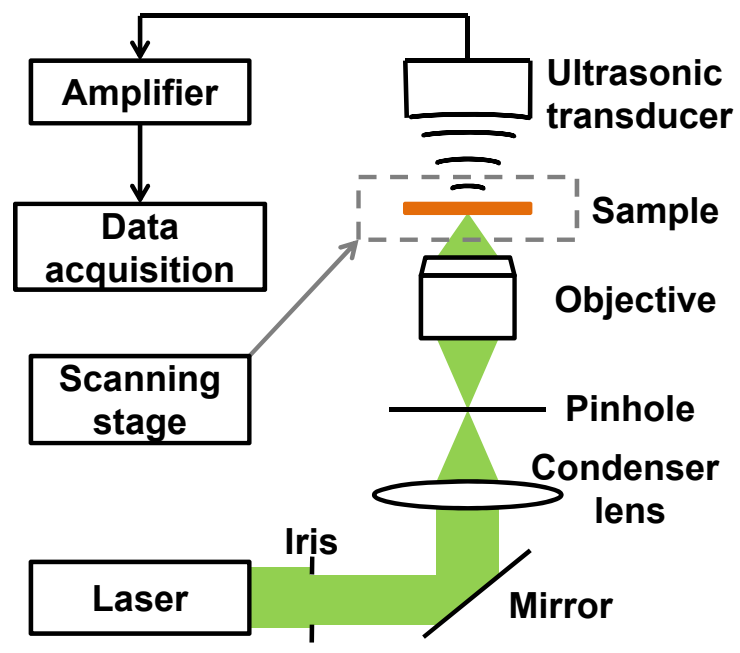

(a)

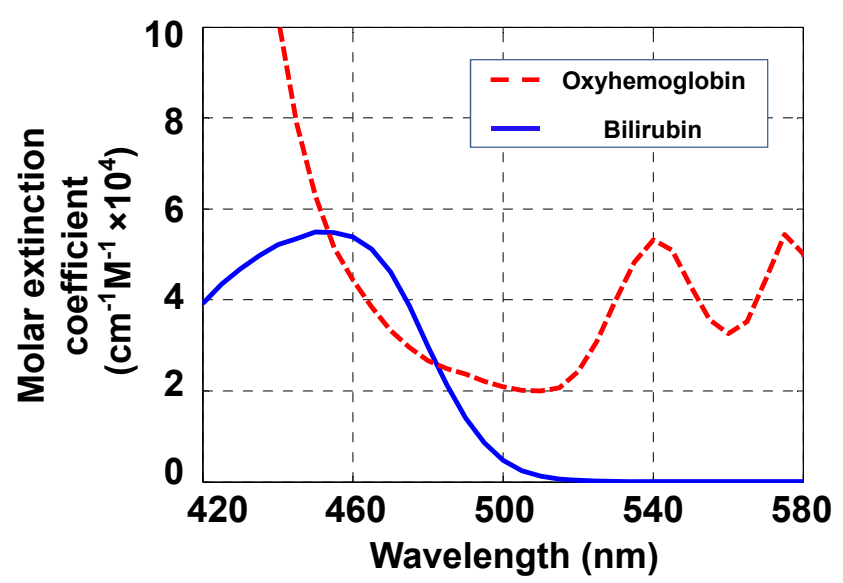

(b)

Fig. 1. (a) Schematic of the PAM system. (b) Absorption spectral of oxyhemoglobin and bilirubin.

Fig. 1(b) shows the absorption spectra of bilirubin and blood ${ }^{30-32}$. Because bilirubin has a relatively high absorption in the wavelength range from $420 \mathrm{~nm}$ to $490 \mathrm{~nm}$, we chose this range for the pure bilirubin measurement. Likewise, because the absorption ratio between bilirubin and blood is relatively high in the wavelength range from $460 \mathrm{~nm}$ to $490 \mathrm{~nm},{ }^{30-32}$ which enables an easier differentiation of bilirubin from blood, we chose this range for the blood mixed sample detection. Also, the wavelength range from $568 \mathrm{~nm}$ to $588 \mathrm{~nm}$ was chosen for blood measurement, where blood has high absorption, but bilirubin has almost no absorption. ${ }^{31-33}$

In the experiments, bilirubin powder (B4126, Sigma-Aldrich) was dissolved in dimethyl sulfoxide solution (Fisher Scientific) and further diluted by water. To prepare the pure tissue-mimicking sample, the bilirubin solution was mixed with 5\% gelatin (G1890, Sigma-Aldrich) and 1\% intralipid (Fresenius Kabi). The congealed sample had an optical scattering coefficient of approximately $100 \mathrm{~cm}^{-1}$. To prepare the mixed sample (to mimic blood vessels), the bilirubin solution was mixed with lysed bovine blood with oxygen (905, Quad Five). The mixture was then contained in a microtube (made of platinum-cured silicone) with an inner diameter of $300 \mu \mathrm{m}$ and an outer diameter of $600 \mu \mathrm{m}(60985-700, \mathrm{VWR})$ to mimic a blood vessel.

\section{Results}

We implemented quantitative PAM of pure bilirubin samples. Five solid samples with different volume-averaged bilirubin concentrations $(1,2,3,4$, and $5 \mathrm{mg} / \mathrm{dL})$ were prepared as described above, which covered the normal and elevated bilirubin levels in humans. Each sample had a cylindrical shape with a diameter of about $500 \mu \mathrm{m}$ (determined by the inner diameter of the metal tube used as the sample mold), and the space between the samples was filled with ultrasonic gel. With a step size of $2 \mu \mathrm{m}$, a $3 \mathrm{~mm}$ by $0.12 \mathrm{~mm}$ area was scanned at $430 \mathrm{~nm}$ wavelength, where bilirubin has a strong absorption to acquire PAM images of bilirubin distributions [Fig. 2(a)]. The volumeaveraged concentrations of bilirubin in the samples were set to be $1 \mathrm{mg} / \mathrm{dL}$ to $5 \mathrm{mg} / \mathrm{dL}$ from the top to the bottom. Here the sample and the background in the image were separated at a threshold of 5 times of the noise level. The measured average concentration of bilirubin in each sample was in accordance with the preset concentration as 
shown in Fig. 2(b). The results show that the PAM system not only can measure the bilirubin concentration but also can provide its spatial information in the pure bilirubin sample.

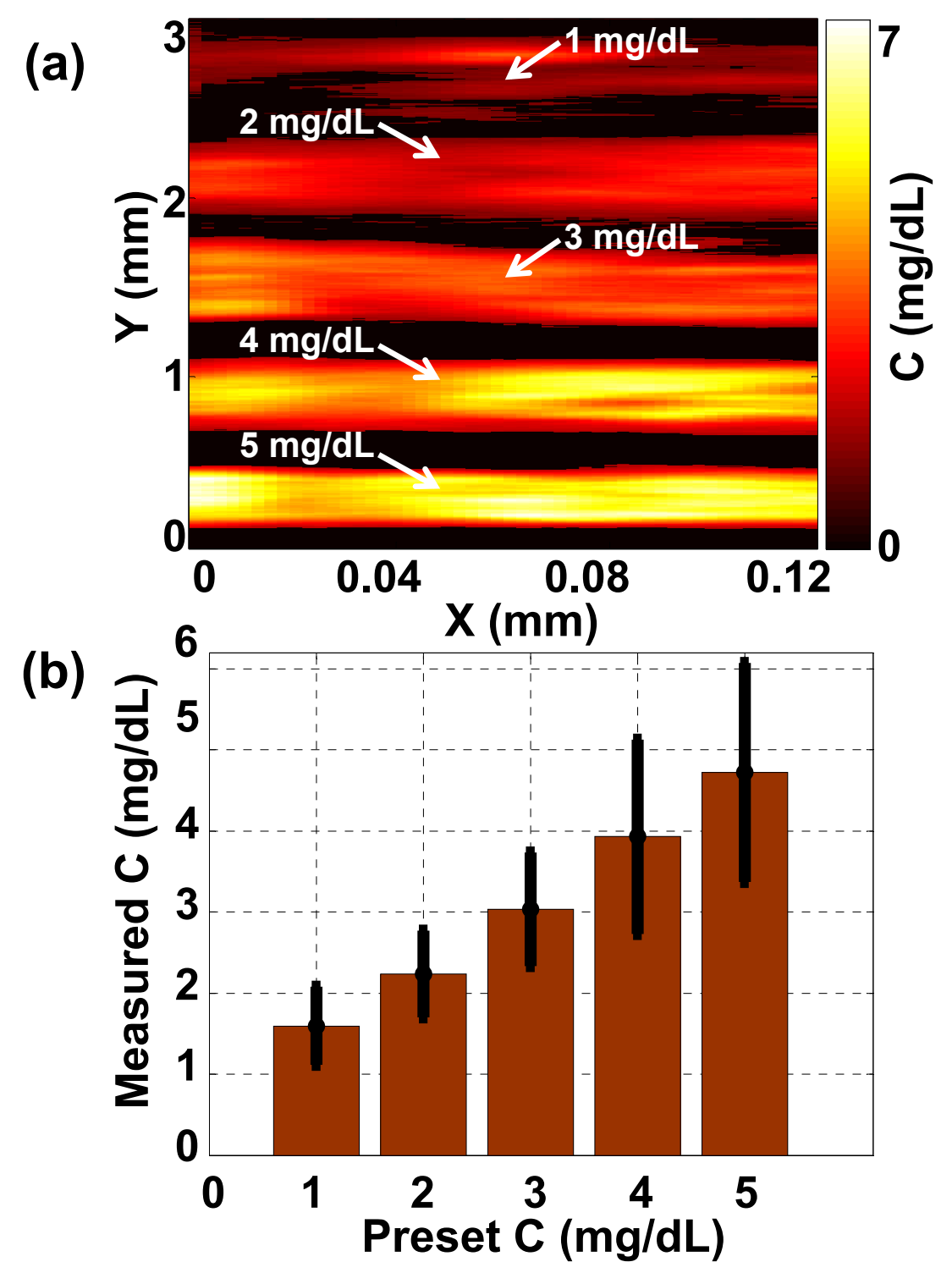

Fig. 2. Quantitative PAM of tissue-mimicking samples containing bilirubin with varied concentrations. (a) Bilirubin distribution image. From top to bottom in the image, the sample concentrations are 1, 2, 3, 4, and $5 \mathrm{mg} / \mathrm{dL}$, respectively. (b) Volumeaveraged bilirubin concentrations from the PAM measurements in each sample.

We also detected bilirubin distribution in the mixed samples. Wavelengths of $460 \mathrm{~nm}, 470 \mathrm{~nm}, 480 \mathrm{~nm}, 490 \mathrm{~nm}$, $570 \mathrm{~nm}$, and $578 \mathrm{~nm}$ were selected. Solutions with bilirubin-to-blood ratio of 2, 4, 6, 8 and $10 \mathrm{mg} / \mathrm{dL}$ to $148 \mathrm{~g} / \mathrm{L}$ were prepared, corresponding to both the normal and the elevated ratios in physiology. Five tubes with the same diameters as in the previous experiment were utilized to hold the mixture samples. Here, a $4 \mathrm{~mm}$ by $0.5 \mathrm{~mm}$ area 
was scanned with a step size of $2 \mu \mathrm{m}$. The image of the bilirubin concentration distribution is shown in Fig. 3(a). The average bilirubin concentrations in the sample from top to bottom were set to be $2 \mathrm{mg} / \mathrm{dL}$ to $10 \mathrm{mg} / \mathrm{dL}$ with respect to the blood concentration of $148 \mathrm{~g} / \mathrm{L}$. The measured average concentrations of bilirubin are in accordance with the preset concentrations, as shown in Fig. 3(b). The results illustrate that PAM can quantitatively image bilirubin distributions in the presence of blood.

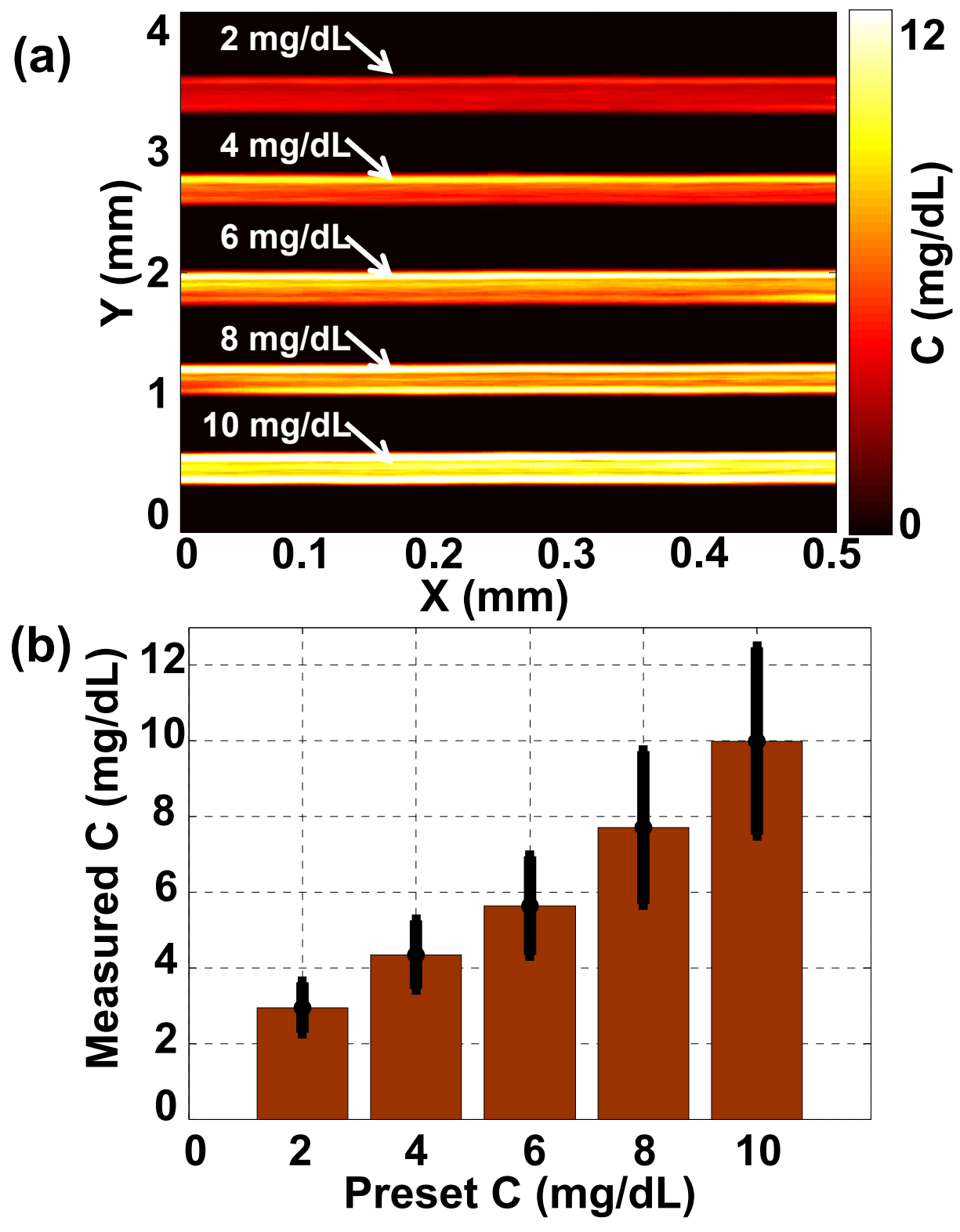

Fig. 3. Multi-wavelength PAM of the mixtures of bilirubin and blood. (a) Quantitative PAM images at six optical wavelengths.

(b) Bilirubin distribution image calculated from the multi-wavelength PAM images. From top to bottom in the image, the bilirubin concentrations are $2,4,6,8$, and $10 \mathrm{mg} / \mathrm{dL}$, respectively, whereas the blood concentration is $148 \mathrm{~g} / \mathrm{L}$. (c) Volumeaveraged bilirubin concentration from the PAM measurements in each tube. 


\section{Conclusion}

In summary, we have demonstrated the ability of PAM for quantitative mapping of bilirubin distribution. The bilirubin distribution both in the pure bilirubin samples and in the blood-mixed samples was imaged by multiwavelength PAM. Therefore, our method shows promise to quantitatively image bilirubin in vivo for further clinical applications, such as the diagnosis of jaundice.

\section{Acknowledgments}

This work was sponsored in part by National Institutes of Health grants R01 EB000712, R01 EB008085, R01 CA134539, U54 CA136398, R01 CA157277, and R01 CA159959. L.W. has a financial interest in Microphotoacoustics, Inc. and Endra, Inc., which, however, did not support this work.

\section{Reference}

[1] S. P. Roche and R. Kobos, "Jaundice in the Adult Patient," Am. Fam. Physician 69, 299-304 (2004)

[2] American Academy of Pediatrics Subcommittee on Hyperbilirubinemia, "Management of hyperbilirubinemia in the newborn infant 35 or more weeks of gestation," Pediatrics 114, 297-316 (2004).

[3] V. K. Bhutani and L. Johnson, "Kernicterus in the 21st century: frequently asked questions," J. Perinatol. 29, S20-S24 (2009).

[4] C.E. Ahlfors, "Criteria for exchange transfusion in jaundiced newborns," Pediatrics 93, 488-494 (1994).

[5] S. N. EI-Beshbishi, K. E. Shattuck, A. A. Mohammad, and J. R. Petersen, "Hyperbilirubinemia and transcutaneous bilirubinometry," Clin. Chem. 55 (7), 1280-1287 (2009).

[6] F. F. Rubaltelli, R. G. Glenn, N. Loskamp, N. Modi, M. R. Kleiner, A. Sender, and P. Vert, "Transcutaneous bilirubin measurement: a multicenter evaluation of a new device," Pediatrics 107, 1264-71 (2001).

[7] L. L. Randeberg, E. Larsen and L. Svaasand, "Characterization of vascular structures and skin bruises using hyperspectral imaging, image analysis and diffusion theory," J. Biophoton. 3 (1-2), 53-65 (2010).

[8] Y. Yamauchi and I. Yamanouchi, "Transcutaneous Bilirubinometry: Bilirubin Kinetics of the Skin and Serum during and after Phototherapy,” Biol. Neonate. 56, 263-9 (1989).

[9] G. Agati, F. Fusi, G.P. Donzelli, and R. Pratesi, "Quantum yield and skin filtering effects on the formation rate of lumirubin," J. Photochem. Photobiol. B: BioZ. 18, 197-203 (1993).

[10] S. L. Jacques, I. Saidi, A. Ladner, and D. Oelberg, "Developing an optical fiber reflectance spectrometer to monitor bilirubinemia in neonates," Proc. SPIE 2975, 115-124 (1997).

[11] S.K. Alla, A. Huddle, J.D. Butler, P.S. Bowman, J.F. Clark, and F.R. Beyette, "Point-of-Care Device for Quantification of Bilirubin in Skin Tissue," IEEE Trans. Biomed. Eng. 58, 3 (2011).

[12] M. J. Maisels, E. M. Ostrea, S. Touch, S. E. Clune, E. Cepeda, E. Kring, K. Gracey, C. Jackson, D. Talbot, and R. Huang, "Evaluation of a new transcutaneous bilirubinometer," Pediatrics 113, 1628-1635 (2004).

[13] G. Bertini, S. Pratesi, E. Cosenza, and C. Dani, "Transcutaneous bilirubin measurement: evaluation of Bilitest," Neonatology 93, 101-105(2008).

[14] G. Payne, N. Langlois, C. Lennard, and C. Roux, "Applying visible hyperspectral (chemical) imaging to estimate the age of bruises," Med. Sci. Law 47 (3), 225-232 (2007). 
[15] K. Maslov, G. Stoica, and L. V. Wang, "In vivo dark-field reflection-mode photoacoustic microscopy," Opt. Lett. 30, 625-627 (2005).

[16] H. F. Zhang, K. Maslov, G. Stoica, and L. V. Wang, "Functional photoacoustic microscopy for high-resolution and noninvasive in vivo imaging," Nat. Biotechnol. 24, 848 (2006).

[17] K. Maslov, H. F. Zhang, S. Hu, and L. V. Wang, "Optical-resolution photoacoustic microscopy for in vivo imaging of single capillaries," Opt. Lett. 33, 929-931 (2008).

[18] L. V. Wang, "Multiscale photoacoustic microscopy and computed tomography," Nat. Photon. 3, 503-509 (2009).

[19] S. Hu and L. V. Wang, "Photoacoustic imaging and characterization of the microvasculature," J. Biomed. Opt. $15(1), 011101(2010)$.

[20] L. V. Wang and S. Hu, "Photoacoustic tomography: in vivo imaging from organelles to organs," Science 335 (6075), 1458-1462 (2012).

[21] L. V. Wang and H. Wu, Biomedical Optics: Principles and Imaging, Wiley, Hoboken, NJ (2007).

[22] J. Yao, K. I. Maslov, Y. Zhang, Y. Xia, and L. V. Wang, "Label-free oxygen-metabolic photoacoustic microscopy in vivo," J. Biomed. Opt. 16(7), 076003 (2011)

[23] E. W. Stein, K. Maslov, and L. V. Wang, "Noninvasive, in vivo imaging of blood-oxygenation dynamics within the mouse brain using photoacoustic microscopy," J. Biomed. Opt. 14, 020502 (2009)

[24] D. K. Yao, K. Maslov, K. K. Shung, Q. F. Zhou, and L. V. Wang, "In vivo label-free photoacoustic microscopy of cell nuclei by excitation of DNA and RNA," Opt. Lett. 35 (24), 4139-4141 (2010).

[25] Z. Xu, C. H. Li, and L. V. Wang, "Photoacoustic tomography of water in phantoms and tissue," J. Biomed. Opt. 15 (3), 036019 (2010).

[26] H. W. Wang, N. Chai, P. Wang, S. Hu, W. Dou, D. Umulis, L. V. Wang, M. Sturek, R. Lucht, and J. Cheng, "Label-free bond-selective imaging by listening to vibrationally excited molecules," Phy. Rev. Lett. 106 (23), 238106 (2011).

[27] C. Zhang, Y. Zhang, D.-K. Yao, Y. Xia, and L. V. Wang, "Label-free photoacoustic microscopy of cytochromes," J. Biomed. Opt. 18(2), 020504 (2013).

[28] Y. Wang, K. Maslov, Y. Zhang, S. Hu, L. Yang, Y. Xia, J. Liu, and L. V. Wang, "Fiber-laser-based photoacoustic microscopy and melanoma cell detection," J. Biomed. Opt. 16, 011014 (2011).

[29] C. Zhang, K. Maslov and L. V. Wang, "Subwavelength-resolution label-free photoacoustic microscopy of optical absorption in vivo", Opt. Lett. 35, 3195-3197 (2010).

[30] J. M. Schmitt, "Optical measurement of blood oxygen by implantable telemetry," Ph.D. Dissertation, Stanford University (1986).

[31] G. Agati and F. Fusi, "New trends in photobiology recent advances in bilirubin photophysics," J. Photochem. Photobiol. 7, 1-14 (1990).

[32] J. M. Dixon, M. Taniguchi and J. S. Lindsey, "PhotochemCAD 2. A Refined Program with Accompanying Spectral Databases for Photochemical Calculations," Photochem. Photobiol. 81, 212-213 (2005).

[33] Y. Zhou, C. Zhang, D.-K. Yao, and L. V. Wang, "Photoacoustic microscopy of bilirubin in tissue phantoms," $J$. Biomed. Opt. 17(12), 126019 (2012). 